\title{
Decay of the vortex tangle at zero temperature and quasiclassical turbulence
}

\author{
Sergey K. Nemirovskii \\ Institute of Thermophysics, Lavrentyev Ave, 1, Novosibirsk 630090, Russia, \\ Novosibirsk State University, Novosibirsk, Russia \\ E-mail: nemir@itp.nsc.ru
}

Received February 21, 2013

\begin{abstract}
We review and analyze a series of works, both experimental and numerical and theoretical, dealing with the decay of quantum turbulence at zero temperature. Free decay of the vortex tangle is a key argument in favor of the idea that a chaotic set of quantum vortices can mimic classical turbulence, or at least reproduce many of the basic features. The corresponding topic is referred as the quasiclassical turbulence. Appreciating significance of the challenging problem of classical turbulence it can be expressed that the idea to study it in terms of quantized line is indeed very important and may be regarded as a breakthrough. For this reason, the whole theory, together with the supporting experimental results and numerical simulations should be carefully scrutinized. One of the main arguments, supporting the idea of quasiclassical turbulence is the fact that vortex tangle decays at zero temperature, when the mutual friction is absent. Since all other possible mechanisms of dissipation of the vortex energy, discussed in literature, are related to the small scales, it is natural to suggest that the Kolmogorov cascade takes place with the flow of the energy in space of scales, just like as in the classical turbulence. In the present work we discuss an alternative mechanism of decay of the vortex tangle, which is not associated with dissipation at small scales. This mechanism is a diffusive-like spreading of the vortex tangle due to evaporation of small vortex loops. We discuss a number of experiments and numerical simulations, considering them from the point of view of alternative mechanism.
\end{abstract}

PACS: $67.25 . d k$ Vortices and turbulence;

47.37.+q Hydrodynamic aspects of superfluidity; quantum fluids;

05.20. $-\mathbf{y}$ Classical statistical mechanics.

Keywords: quasiclassical turbulence, zero temperature, Kolmogorov turbulence.

\section{Introduction and scientific background}

Decay of quantum turbulence at zero temperature is one of the puzzling and fascinating phenomenon of the quantized vortices dynamics. Indeed, in this case, the mutual friction is absent, and there is no apparent mechanism of dissipation of the vortex tangle. Nevertheless, the authors of both experimental works (see, e.g., [1-4]) and of numerical simulations (see, e.g., [5,6]) report on attenuation of the vortex line density at a very low temperature. The low temperature decay of the vortex tangle is closely related to the hypothetical connection between the classical (Kolmogorov) and quantum turbulence. The idea that the chaotic set of quantum vortices can mimic classical turbulence, or at least reproduce many of the basic features, is currently actively being developed. The exhaustive material can be found in a series of reviews that has recently appeared; we would like to draw the reader's attention to papers [7-12], and to papers included into special issue of JLTP [13] and into book [14]. In principle, this idea had been discussed early (see, e.g., famous textbook by Frisch [15]), as an alternative version of the problem of classical turbulence. But only now, when the new powerful experimental methods in quantum fluids appeared, this idea can be checked experimentally and it seems to be very attractive. Appreciating significance of the challenging problem of the classical turbulence it can be expressed that the idea to study it in terms of quantized line is indeed very important and may be regarded as a breakthrough. For this reason, this theory should be carefully scrutinized.

One of the basic arguments supporting the idea of Kolmogorov turbulence in quantum fluids is the fact that vortex tangle decays at zero temperature, when the apparent mechanism of dissipation (mutual friction) is absent. The physical mechanisms of the dissipation can be various, 
some approaches and ideas such as a cascade-like breakdown of the loops, Kelvin waves cascade, acoustic radiation, reconnection loss, etc., have been discussed in detail in recent reviews $[11,12,16]$. It is remarkable that all of these mechanisms are realized only on a very small scale. Therefore, it is natural to suggest that the Kolmogorov cascade occurs with the flow of energy, just as in the classical turbulence. The mentioned experimental works on the decay of the vortex tangle were interpreted on the basis of the decay of the classical turbulence. In works by Skrbek [17] it was developed approach, which relates the attenuation of the energy in classical turbulence to the decay of the vortex line density and predicts the temporal dependence of this attenuation.

In the present work we review and analyze the both experimental works and numerical simulations, dealing with the decay of the quantum turbulence at zero temperature. The discussion is grounded on the alternative mechanism of decay of the vortex tangle, which is not related to dissipation at small scales. This mechanism is the diffusive-like spreading of the vortex tangle, connected to evaporation of small vortex loops. We applied the diffusion equation obtained early ([18]) to describe the decay of superfluid turbulence in the listed above experiment. Our calculations enable us to conclude that mechanism of diffusion can describe correctly the attenuation of the vortex line density. Besides, we discuss these experiments from position of theory Kolmogorov turbulence.

\section{Diffusion equation}

In this section we very briefly describe main ideas leading to the diffusion theory of the vortex loops, details can be found in paper [18]. Vortex loops composing the vortex tangle can move as a whole with some drift velocity $V_{l}$ depending on their structure and their length. The flux of the line length, energy, momentum etc., executed by the moving vortex loops takes place. In the case of inhomogeneous vortex tangle the net flux $\mathbf{J}$ of the vortex length due to the gradient of concentration of the vortex line density $\mathcal{L}(x, t)$ appears. The situation here is exactly the same as in classical kinetic theory with the difference being that the "carriers" are not the point particles but the extended objects (vortex loops), which possess an infinite number of degrees of freedom with very involved dynamics. We offer to fulfill investigation basing on the supposition that vortex loops have the Brownian or random walking structure with the generalized Wiener distribution (see [19-21]).

To develop the theory of the transport processes fulfilled by vortex loops (in spirit of classical kinetic theory) we need to calculate the drift velocity $V_{l}$ and the free path $\lambda(l)$ for the loop of size $l$. Referring to the paper [18] we write down here the following result. The drift velocity $V_{l}$ for the loop of size $l$ is

$$
V_{l}=C_{v} \beta / \sqrt{l \xi_{0}} .
$$

Quantity $\beta$ is $(\kappa / 4 \pi) \ln \left(\mathcal{L}^{-1 / 2} / a_{0}\right)$, where $\kappa$ is the quantum of circulation and $a_{0}$ is the core radius, $C_{v}$ is numerical factor of the order of unity. The $\xi_{0}$ is the parameter of the generalized Wiener distribution, it is of order of the interline space $\mathcal{L}^{-1 / 2}$. The free path $\lambda(l)$ for loop of length $l$ is

$$
\lambda(l)=1 / 2 l b_{m} \mathcal{L}
$$

Here, $b_{m}$ is the numerical factor, approximately equal to $b_{m} \approx 0.2$. It is seen that free path $\lambda(l)$ is very small, it implies only very small loops give a significant contribution to transport processes. Knowing the averaged velocity $V_{l}$ of loops, and the probability $P(x)$ (both quantities are $l$-dependent), we can evaluate the spatial flux of the vortex line density $\mathcal{L}$, executed by the loops. The procedure is very close to the one in the classical kinetic theory, with the difference being that the carriers have different sizes, requiring additional integration over the loop lengths. Referring again to paper [18] we write the flux $\mathbf{J}$ of vortex line is proportional to $\nabla \mathcal{L}$ and, correspondingly, the spatial-temporal evolution of quantity $\mathcal{L}$ obeys the diffusion type equation

$$
\frac{\partial \mathcal{L}}{\partial t}=D_{v} \nabla^{2} \mathcal{L}
$$

Our approach is a fairly crude to claim a good quantitative description. However, if we are to adopt the data grounded on the exact solution to the Boltzmann type "kinetic" equation for vortex loops distribution ([21]), we conclude that $C_{d} \approx 2.2$. Further we use the (3) to describe the decay of superfluid turbulence in various experiments including numerical simulations.

\section{Revision of the experimental data and numerical simulations}

In this section we discuss several experiments and numerical simulation on the decay of the vortex, which are usually considered as the ground for the Kolmogorov decay of the superfluid turbulence. They are the so called Lancaster experiment [3], the Manchester experiment [4], the Osaka numerical simulation [5] and the Novosibirsk numerical simulation [6].

\subsection{Lancaster experiment}

Let us now discuss the recent experiment on decay of the vortex tangle at very low temperatures [3]. The authors of this work reported the attenuation of vortex line density in superfluid turbulent helium, ${ }^{3} \mathrm{He}-B$. In the upper picture of Fig. 1, we displayed Fig. 2 of work [3], showing results of measurements on the temporal behavior of the average vortex line density $\mathcal{L}(t)$, (solid curves, see [3] for details). Authors compare their data with the theory by Skrbek [17] and conclude that decay of the vortex tangle occurs in accordance with theory of classical (Kolmogorov) turbu- 

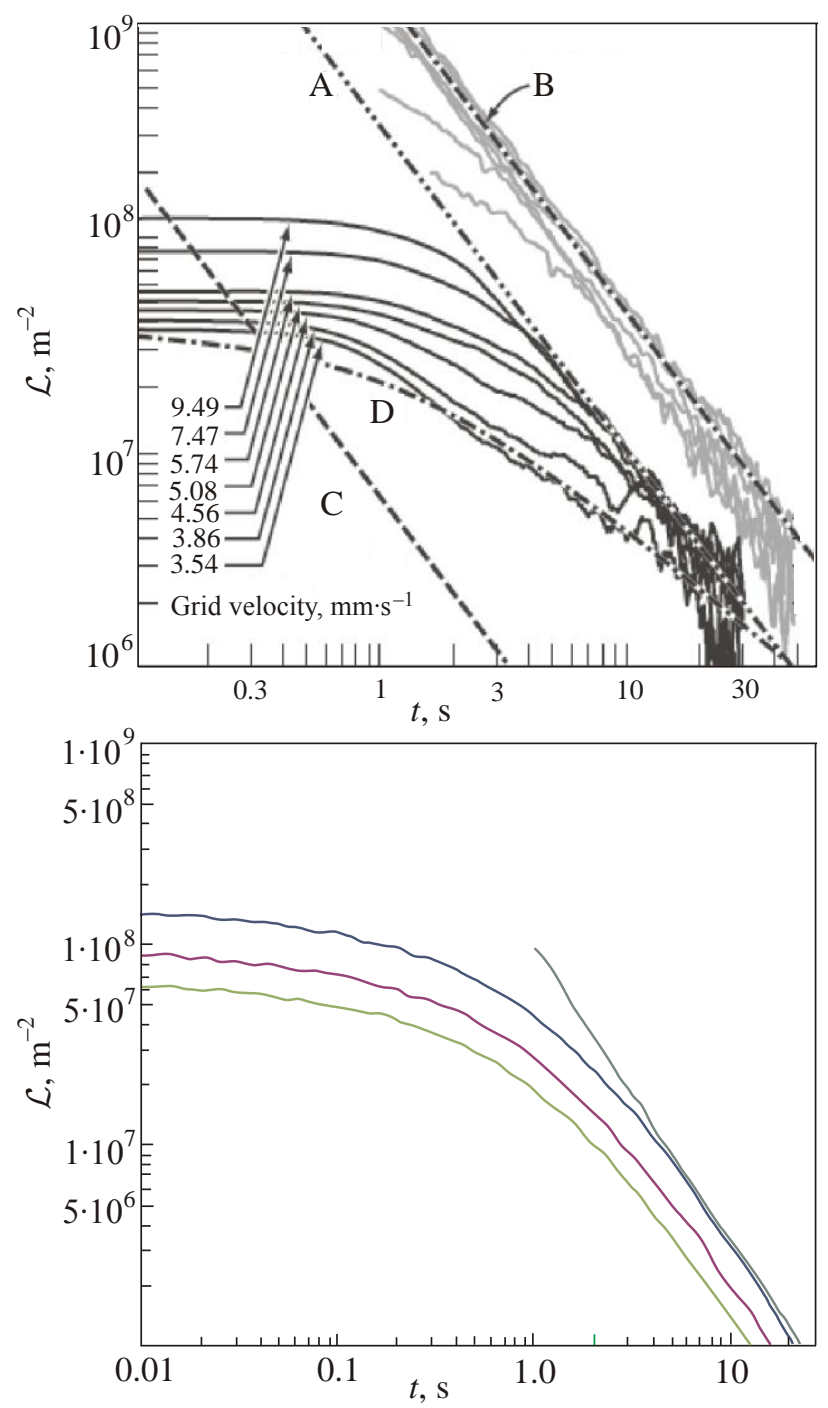

Fig. 1. Comparison with experiment [3]. In the upper picture we displayed Fig. 2 of work [3], showing results of measurements on the temporal behavior of the average vortex line density $\mathcal{L}(t)$, (solid curves, see [3] for details). We calculated the same quantity resolving the diffusion Eq. (3), with the use the boundary condition, which corresponds to the smearing of the vortex tangle into ambient space. It is known that for this condition the solution of three-dimensional problem is just production of three onedimensional solutions. The straight line in the lower picture exactly corresponds to line $\mathrm{A}$, in the upper picture (this line was named by the authors of paper [3] as a "limiting behavior").

lence. The main argument is that the long time attenuation of the vortex length is described by the same line A ("limiting behavior") with the $t^{-3 / 2}$ dependence (almost independently on initial level). The according kinematic viscosity is $v^{\prime} \sim 0.2 \kappa$.

It is necessary to note the following circumstance. The one of the standard vision how the set of the vortex filament can imitate the classical turbulence is that the lines are unified into the bundles (containing many lines). The set of many bundles of various sizes randomly move, imitating the dynamics of eddies in classical turbulence. Other view is that in the dense vortex tangle there is polarization "indistinguishable by glance" and these polarized vortices also reproduce the eddy dynamics. It is necessary to note the following circumstance. The one of the standard vision how the set of the vortex filament can imitate the classical turbulence is that the lines are unified into the bundles (containing many lines). The set of many bundles of various sizes randomly move, imitating the dynamics of eddies in the classical turbulence. Other view is that in the dense vortex tangle there is an averaged partial polarization of lines "indistinguishable by glance" and these polarized vortices also reproduce the eddy dynamics. Let us consider the situation in the Lancaster experiment more attentively. Let us take some "intermediate" value of the vortex line density $\mathcal{L}(t)=0.5 \cdot 10^{3} \mathrm{~cm}^{-2}$, where all lines are collapsed into the "limiting", universal behavior (line " $A$ " in the upper picture of Fig. 1). For this value the interline space is equal to $\mathcal{L}^{-1 / 2} \approx 4.5 \cdot 10^{-2} \mathrm{~cm}$. The latter implies that in the volume with size $d=1.5 \mathrm{~mm}$ (offered by authors as the region, where the vortex tangle evolves) we have approximately $d / L^{-1 / 2} \approx 3$ lines. Of course, this amount is not enough to form many bundles with very "dense array of vortex lines", which are necessary to "mimic classical turbulence". On the same ground one can assert that it is hardly possible to say about the partial polarization of lines in the dense tangle "indistinguishable by glance".

Another fact is that about one third of the "limiting line A" is occupied by the developed fluctuations, which can blur the true dependence.

Consequently supposing that the decay is realized by the escaping of the vortex loops we had estimated the contribution into attenuation of the vortex line density, due to the pure diffusion mechanism. We use the classical solution (see for details [18]). Results (with comments) are depicted in Fig. 1. We again can conclude that the diffusion spreading describes satisfactorily the evolution of the vortex tangle, without any additional mechanism.

Concluding this subsection we again can state that (i) interpretation of experiment is not fully consistent and cannot serve as convincing evidence in favor of existence of cascade-like transfer of the vortex length (and energy)in direction of small scales, and (ii) decay of the superfluid turbulence is quite well described by the diffusion type mechanism.

\subsection{Manchester experiment}

The next experiment which we would like to discuss and which is also frequently referred as the evidence of the Kolmogorov turbulence imitated by quantized vortex lines is the work [4]. In this work the decay of vortex tangle in He II was observed in the closed cube with solid walls. Results are collected in the upper picture of Fig. 2, where the temporal behavior of the average vortex line density $\mathcal{L}_{\mathrm{av}}(t)$ is depicted. Authors compare their data with the 

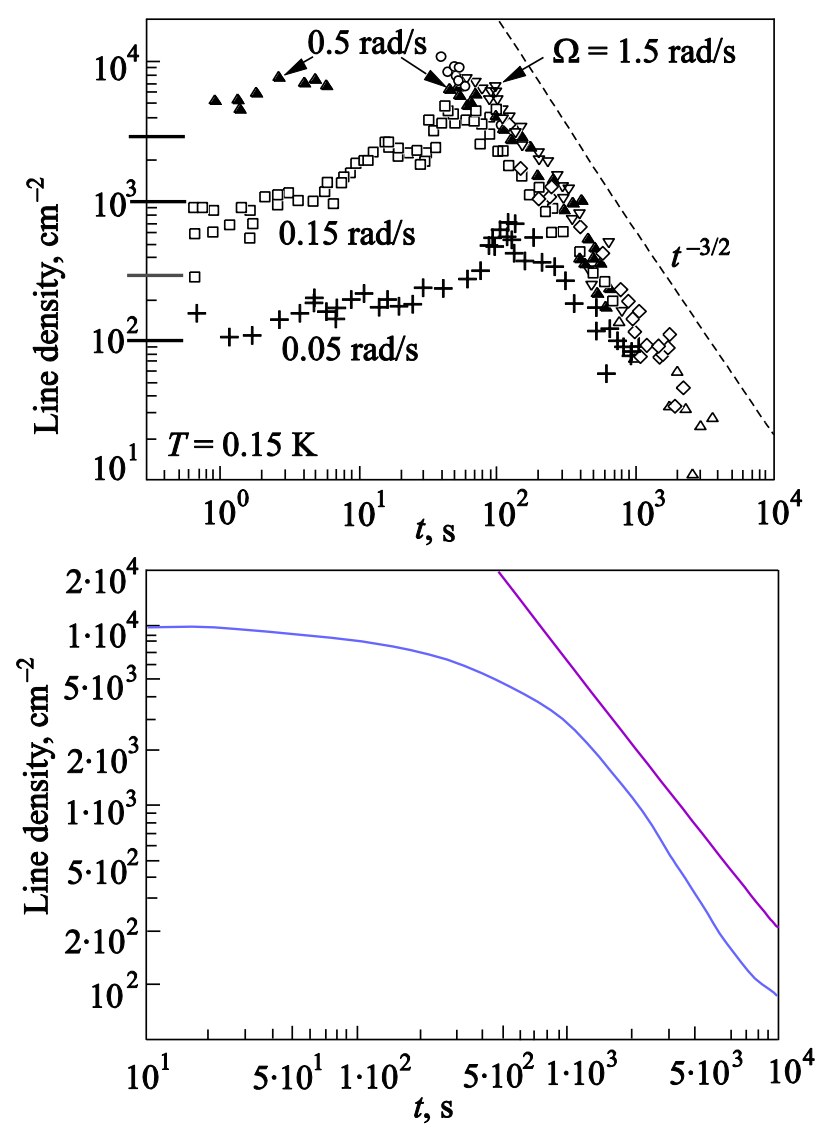

Fig. 2. Comparison with experiment [4]. The temporal behavior of the average vortex line density $\mathcal{L}_{\text {av }}(t)$ obtained in [4] is depicted. We calculated the same dependence on the basis of the diffusion Eq. (3), with the boundary accounting back radiation of loops from the solid walls. The full three-dimensional problem had been resolved numerically the result shown in the low picture.

theory by Skrbek [17] and conclude that decay of the vortex tangle occurs in accordance with theory of classical (Kolmogorov) turbulence. The according kinematic viscosity is $v^{\prime} \sim 0.003 \kappa$, which about two orders smaller, than obtained in the Lancaster experiment. Authors of [4] assumed that the source of this discrepancy is that the turbulence observed in [3] is not homogeneous, and the size of the energy-containing eddies may differ from the spatial extent of the turbulence, so that the value of $v^{\prime}$ obtained in [3] was ambiguous.

Coming to the previous subsection we can say that situation with the number of vortices is better than in the Lancaster experiment. It is should be noted however that it is merely due to larger size of the experimental cell. If to take parts of experimental cell with sizes of the order $3 \mathrm{~mm}$ we again meet the of very dilute vortex tangle. Another remark is related to the Volovik observation [22]. At the low temperature in ${ }^{3} \mathrm{He}-B$ and almost at any temperature in ${ }^{4} \mathrm{He}$, there should the so called Vinen (not Kolmogorov) turbulence, and, the whole ideology grounded on the Skrbek theory is not applicable in the case of Manchester experiment. In our opinion the large difference in the value of kinematic viscosity is $v^{\prime}$ in works [3] and [4] (in fact it is just difference in the total time of the decay) is related to (i) the different sizes of the cells, where the superfluid turbulence is activated, and (ii) to the different boundary conditions (solid walls in [4], and absence of boundaries in [3]). These two facts definitely point out that the decay has the diffusive nature. We calculated the decay of the vortex tangle on the basis of the diffusion Eq. (3). The fully three-dimensional problem was resolved numerically (see for details [18]), the result shown in the lower picture of Fig. 2. It can be seen that the decay of the vortex tangle, due to diffusion, describes both quantitatively and qualitatively the features observed in the experiment. First of all, the whole qualitative behavior of lines agrees with diffusive-like attenuation. In particular, there is a plateau, which is changed with the fast decay of the tangle. Full decay of the superfluid turbulence occurs in times, which are in a very good agreement, predicted on the basis of the diffusion approach elaborated here. The slope of the curve in the interval of the most intensive decrease, shows the dependence close to $\sim t^{-3 / 2}$, which is also typical for diffusion.

Resuming this subsection we again claim that (i) experiment is not fully self-consistent and (ii) the diffusion mechanism describes well the attenuation of the vortex line density.

\subsection{Osaka numerical simulation}

Results of numerical simulation on the dynamics of the vortex tangle at zero temperature made in Osaka [2], are frequently considered as a base for conclusion that for zero temperature decay of the vortex tangle occurs via various mechanisms realizing at small scales. Attenuation of the vortex line density obtained in numerical simulation [5] is depicted in the upper picture of the Fig. 3.

Let us briefly analyze the situation presented in paper [2], and demonstrate that none of the currently discussed mechanisms of the "homogeneous" decay of the vortex tangle at zero temperature, can be applied to this work. Thus the Kelvin waves cascade could not be a reason for the vortex tangle decay in numerical simulation [5], simply because the space resolution $\Delta \xi=2 \cdot 10^{-2} \mathrm{~cm}$ was too large to monitor the region of large wave numbers, required for observation of a generation of higher harmonics. Similarly, the acoustic radiation could not be a reason for the vortex tangle decay, because the compressibility had not been included in the governing equations, and correspondingly was absent in the numerical scheme. As for the loss of the line length during reconnection, the real effect of the length loss can be obtained only on the basis of more rigorous theory, e.g., with the use of the Gross-Pitaevskii equation. It is known, however, that an artificial loss of length is possible, due to realization of the reconnection procedure. This effect, however, should depend on the space resolution, whereas it was proven that the rate of 

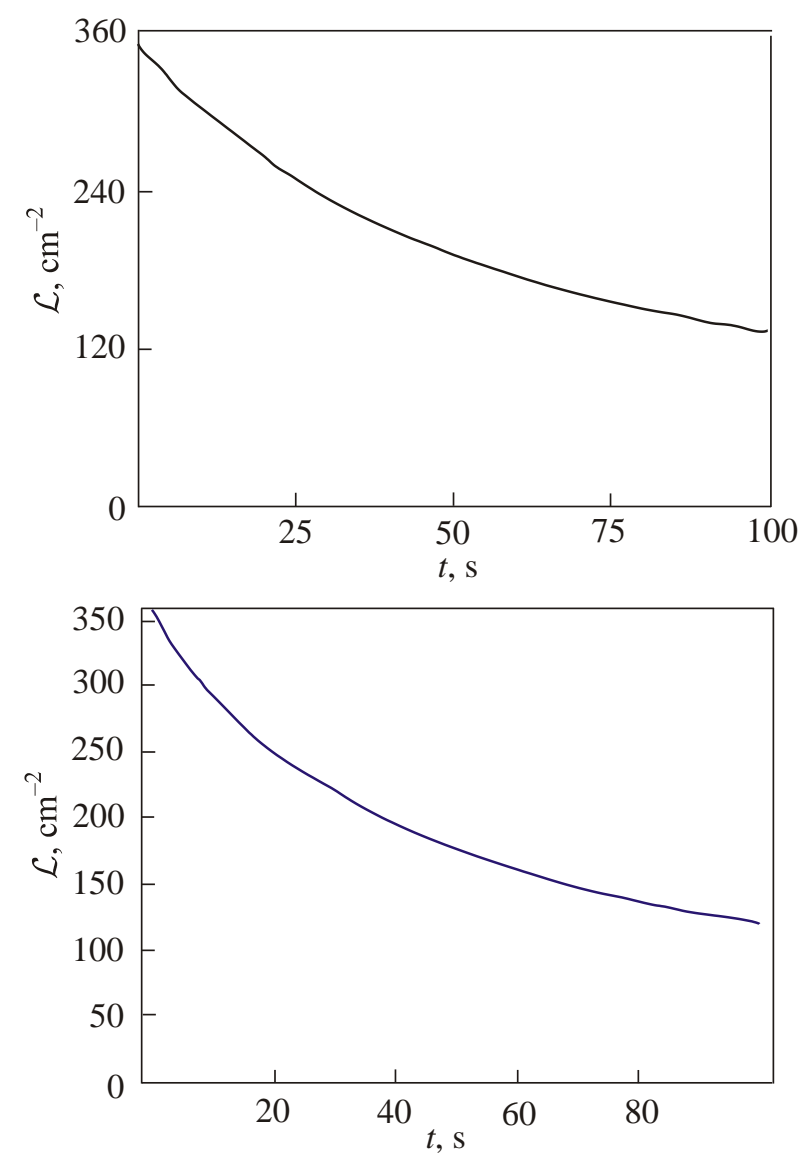

Fig. 3. Comparison with the numerical simulation by Tsubota et al. [2]. In the upper picture it is depicted the attenuation of vortex line density obtained in numerical experiment [2]. In the lower picture it is depicted the same quantity calculated with the use of Eq. (3) without the auxiliary term, the diffusion constant was equal to $D_{v} \approx 2.2 \cdot 10^{-3} \mathrm{~cm}^{2} / \mathrm{s}$. We calculated the twodimensional evolution of the vortex line density in the $1 \mathrm{~cm}$ square resolving numerically Eq. (3) with the boundary conditions accounting the back radiation from the solid walls (see for details [18]).

decay did not depend on it. Feynman cascade of consequent breaking down of vortex loops was imitated in [5] with elimination of very small loops (with sizes of few $\Delta \xi)$. But the total amount of these events was is too small to describe the decay of vortex line density, observed in the numerical experiment.

To resume, one concludes that none of the discussed mechanisms could be the reason of the "homogeneous decay" of the vortex tangle in numerical simulation [5]. Thus, the nature of attenuation of the vortex line density in [5] remained unclear. The only mechanism capable of explain the decrease of the vortex line density $\mathcal{L}(t)$ is just spreading of the tangle and escaping of the vortex loops from the bulk of helium. To check our supposition, we calculated the evolution of the vortex line density under conditions taking in work [5] resolving Eq. (3). The problem had been resolved numerically, the result is depicted in the lower picture of Fig. 3. Comparison of the upper and lower pic- tures of Fig. 3 enabled us to conclude that the diffusion spreading describes satisfactorily the evolution of the vortex tangle, without any additional mechanism.

Resuming this subsection we can state that (i) there is no convincing evidence in favor of existence of cascadelike transfer of the vortex length (and energy) in direction of small scales and (ii) decay of the superfluid turbulence is quite well described by a diffusion mechanism.

\subsection{Novosibirsk numerical simulation}

The direct numerical simulations of the evolution of the nonuniform vortex tangle, originally concentrated in restricted domain, at zero temperature was fulfilled in work [6]. The numerical simulation is performed both with the use of local induction approximation (LIA) and on the base of the Full Biot-Savart law. The purpose of this study was to ascertain the role of various factors arising in the numerical procedure. It is important to stress that the widely accepted mechanism - the cascade-like transferring of the energy by nonlinear Kelvin waves (and radiation of sound) - was not considered. One of the reasons is that the space resolution along the lines did not allow to detect generation of high harmonics, moreover, to get harmonics, which really radiate sound. In addition, the use of the method assumes that the fluid is incompressible.

Numerical simulations have been performed for the cubic domain with transparent walls embedded in an unbounded space, and for the cube with the solid smooth walls. The calculations showed that in the case of unlimited space of the decay of quantum turbulence caused by the evaporation of vortex loops. Result of the numerical simulations is depicted in Fig. 4, it is clearly seen how the vortex loops escape from the volume and vortex line density diminishes inside the bulk. The authors were carefully monitoring all mechanisms leading to the loss of the
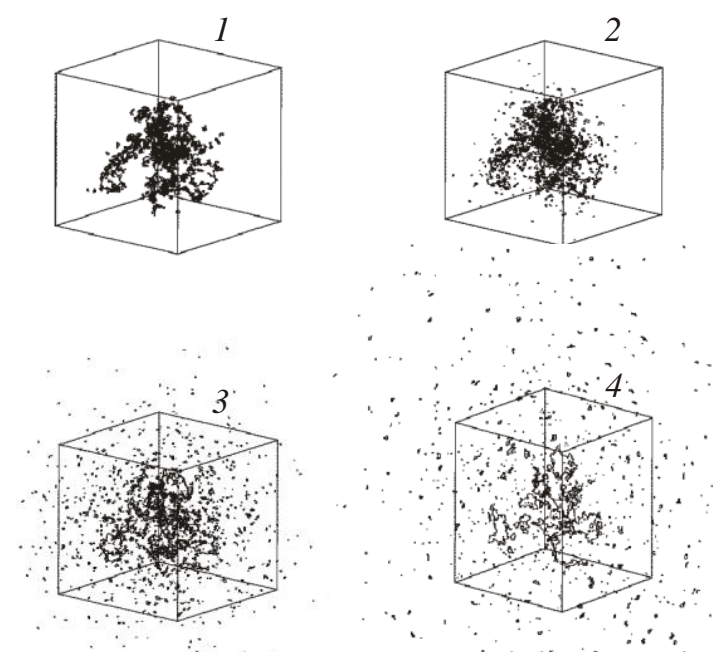

Fig. 4. (Color online) The vortex loops configuration at different moments of times $t$, ms: 0 (1), 0.48 (2), 2.16 (3), 8.63 (4). 
length, such as the change of length owing to reconnection processes, the eliminations of very small loops below the space resolution, the change of length due insertion and removing of points to supply numerical algorithm stability and etc. Results of this monitoring are depicted in Fig. 5. From this figure it can be concluded, that the master mechanism of decrease of the vortex length inside initial volume is related to the escaping of vortex loops

A comparison of the results of the numerical simulations with the theory of diffusion of the vortex tangle described in the Sec. 2 had been made. It was obtained,that the evolution of the length in initial domain is satisfactory described with the diffusion Eq. (3). Result of comparison is depicted in Fig 6. The good agreement with the experimental data and numerical simulation enables us to conclude that the diffusion process plays a dominant role in the free decay of the vortex tangle in the absence of the normal component.

\section{Conclusion}

Our main conclusion would be formulated as follows. The both experimental and numerical data on decay of the superfluid turbulence discussed in the literature cannot be regarded as the firm evidence for the classical turbulence mechanism, accompanied by the Kolmogorov cascade of

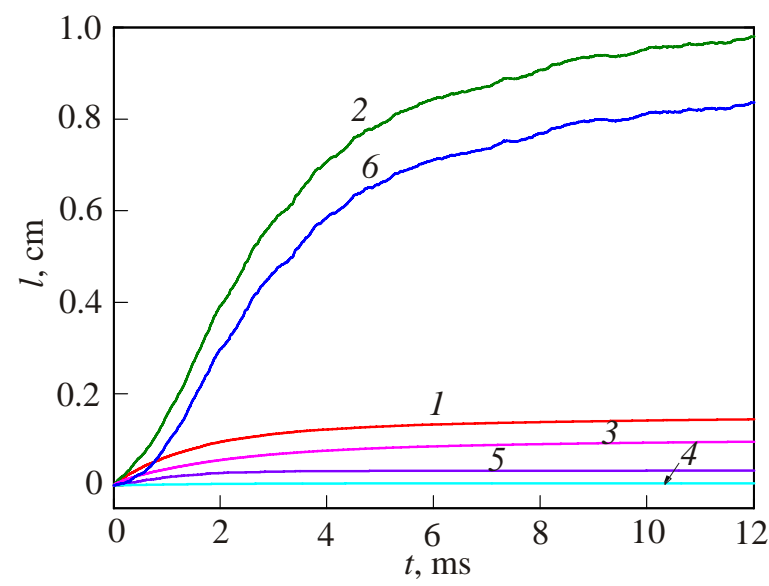

Fig. 5. (Color online) Contribution of various mechanisms into decrease of total length. Decrease of the total length (1), decrease of the total length inside domain (spherical domain with the radius $R=0.008 \mathrm{~cm}$ ) (2), decrease of the total length due to elimination of small loops ( 3 mesh sizes) (3), change of the total length due to artificial procedure - inserting or removing of intermediate points (4), change of the total length due to the reconnection procedure (5), the total length outside domain (6). It is clearly seen that the leading mechanism in decrease of the vortex length inside domain is the escaping of loops. Decrease of the total length $l_{1}$ is equal to sum of all "irreversible" (without escape) mechanisms, $l_{1}=l_{3}+l_{4}+l_{5}$. It is also seen that length outside domain $l_{6}$ plus all "irreversible" losses $l_{1}$ is equal to decrease $l_{2}$ inside domain.

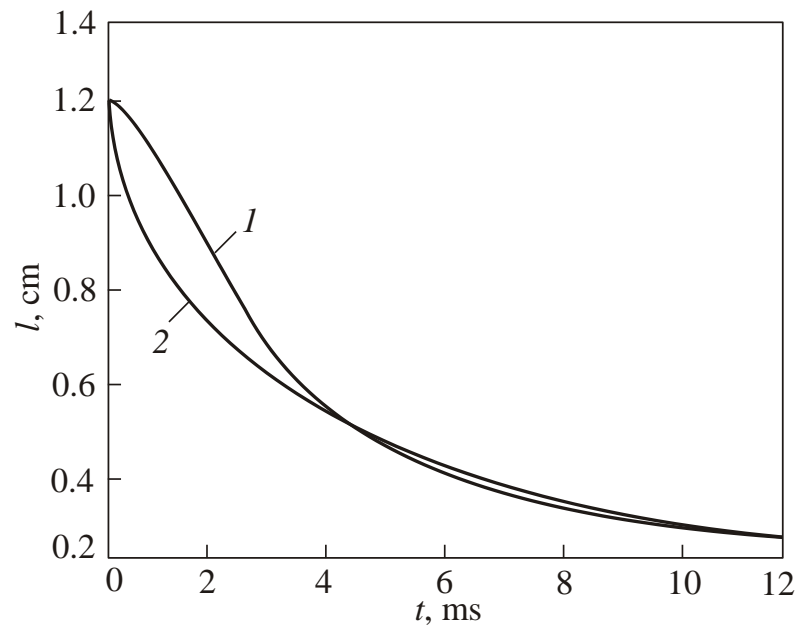

Fig. 6. (Color online) The length $l(t)=\int L(r, t) d r$ inside domain obtained in numerical simulation [6] the total length (curve 1), the same quantity calculated on the base of the diffusion Eq. (3) (curve 2).

the energy to region of very small scales. As we demonstrated, they are not fully self-consistent even in the frame of the accepted approach. At the time we had shown that all experimental data on decay of the vortex tangle agree well with the diffusion mechanism without any additional assumptions. Numerical results confirm this point of view, demonstrating that all possible mechanisms of the loss of the vortex length (and, correspondingly, the vortex energy) are considerably less than the loss of energy due to escaping of the loops from the volume.

\section{Acknowledgments}

The work was supported by the grants No. 10-08-00369 and No. 10-02-00514 from the Russian Foundation of Basic Research, and by the grant from the President Federation on the State Support of Leading Scientific Schools NSh-6686.2012.8.

1. S.I. Davis, P.C. Hendry, and P.V.E. McClintock, Physica B 280, 43 (2000).

2. M. Tsubota, T. Araki, and S.K. Nemirovskii, Phys. Rev. B 62, 11751 (2000).

3. D.I. Bradley, D.O. Clubb, S.N., AM. Guénault, R.P. Haley, C.J. Matthews, G.R. Pickett, V. Tsepelin, and K. Zaki, Phys. Rev. Lett. 96, 035301 (2006).

4. P.M. Walmsley, A.I. Golov, H.E. Hall, A.A. Levchenko, and W.F. Vinen, Phys. Rev. Lett. 99, 265302 (2007).

5. M. Tsubota, T. Araki, and W.F. Vinen, Physica B 329-333, 224 (2003).

6. L. Kondaurova and S.K. Nemirovskii, Phys. Rev. B 86, 134506 (2012).

7. W.F. Vinen, Phys. Rev. B 61, 1410 (2000).

8. W.F. Vinen and R.J. Donnelly, Phys. Today 60, 43 (2007).

9. A. Golov and P. Walmsley, J. Low Temp. Phys. 156, 51 (2009). 
10. W. Vinen, J. Low Temp. Phys. 161, 419 (2010).

11. L. Skrbek and K.R. Sreenivasan, Phys. Fluids 24, 011301 (2012).

12. S.K. Nemirovskii, Phys. Rep. 524, 85 (2013).

13. E.V. Kozik and B.V. Svistunov, J. Low Temp. Phys. 156, 215 (2009); DOI: 10.1007/s10909-009-9914-y.

14. Progress in Low Temperature Physics: Quantum Turbulence, M. Tsubota (ed.), Elsevier Science Publishing Company (2009).

15. U. Frisch, Turbulence, Cambridge University Press, Cambridge (1995).
16. C.F. Barenghi, Physica 237, 2195 (2008).

17. S.R. Stal, L. Skrbek, and R.J. Donnelly, Phys. Rev. Lett 82, $4831(199)$

18. S.K. Nemirovskii, Phys. Rev. B 81, 064512 (2010).

19. S.K. Nemirovskii, Phys. Rev. B 57, 5972 (1998).

20. S.K. Nemirovskii, Phys. Rev. Lett. 96, 015301 (2006).

21. S.K. Nemirovskii, Phys. Rev. B 77, 214509 (2008).

22. G.E. Volovik, JETP Lett. 7, 553 (2004). 